\title{
Levonorgestrel intrauterine system versus thermal balloon ablation for the treatment of heavy menstrual bleeding: A meta-analysis of randomized controlled trials
}

\author{
BING-QING YANG, JIE-HAN XU and YIN-CHENG TENG \\ Department of Obstetrics and Gynecology, Shanghai Jiao Tong University, \\ Affiliated Sixth People's Hospital, Shanghai 200233, P.R. China \\ Received December 16, 2014; Accepted August 13, 2015
}

DOI: $10.3892 /$ etm.2015.2733

\begin{abstract}
At present, there have been no standard research outcomes as to whether the levonorgestrel intrauterine system (LNG-IUS) or thermal balloon ablation (TBA) is superior for the treatment of patients suffering from heavy menstrual bleeding (HMB). Therefore, in the present study, a meta-analysis of randomized controlled trials (RCTs) was conducted in order to compare the effectiveness and affordability of the LNG-IUS with TBA in the treatment of HMB. A literature search of the following electronic databases was conducted: PubMed, EMBASE, the Cochrane Library, Google Scholar, the Chinese Scientific Journals Database, and the China National Knowledge Infrastructure; and a statistical analysis was performed using RevMan 5.2 software. Seven RCTs involving 467 patients (235 LNG-IUS, 232 TBA) met the inclusion criteria for the present study. As assessed by pictorial blood loss assessment chart (PBAC) scores, the LNG-IUS significantly reduced menstrual bleeding after 24 months [standardized mean difference (SMD), -0.86; $95 \%$ confidence interval $(\mathrm{CI}),-1.22$ to $-0.50 ; \mathrm{P}<0.00001]$. Furthermore, the total treatment cost of the LNG-IUS was lower than that of TBA (SMD, -2.35 ; 95\% CI, -2.98 to -1.72 ; $\mathrm{P}<0.00001)$. However, at the 24 month follow-up, side effects such as amenorrhea occurred more frequently in patients treated with the LNG-IUS, as compared with TBA (relative risk, 2.49; 95\% CI, 1.46-4.25; $\mathrm{P}=0.0008)$. No significant differences in hemoglobin levels and quality of life were demonstrated between the two treatment groups. The results of the present meta-analysis suggest that the LNG-IUS may be more effective and affordable than TBA as a long-term treatment (24 months) for HMB. However, following 12-24 months
\end{abstract}

Correspondence to: Professor Yin-Cheng Teng, Department of Obstetrics and Gynecology, Shanghai Jiao Tong University, Affiliated Sixth People's Hospital, 600 Yishan Road, Shanghai 200233, P.R. China

E-mail: teng_yc1008@yeah.net

Key words: heavy menstrual bleeding, levonorgestrel intrauterine system, thermal balloon ablation, meta-analysis of treatment, side effects such as amenorrhea may be more frequent in patients treated with the LNG-IUS. When considering short-term treatment for HMB, controversy remains regarding the two methods and further studies are required to precisely evaluate the outcomes.

\section{Introduction}

Heavy menstrual bleeding (HMB), also known as menorrhagia, is defined as excessive cyclical menstrual bleeding (>80 ml) per menstrual cycle (1). HMB has an enormous impact on women's health and quality of life; therefore, it is unsurprising that HMB is estimated to be the third major cause of visits to gynecology outpatient clinics. Medical therapy is currently the standard treatment for menorrhagia, however it is not always effective. The overall post-operative complication rate is $\sim 9 \%$, of which $1 \%$ of cases are considered to be severe, with a mortality rate of 0.38 per 1,000 patients (2). When other treatments are unsuccessful, a hysterectomy may be performed (3). However, there are various alternatives, such as the levonorgestrel intrauterine system (LNG-IUS) and thermal balloon ablation (TBA), and these second line therapies for menorrhagia may offer potential advantages, including: Reduced morbidity, shorter recovery times, lower cost, fewer serious complications, and an earlier return to routine activities (3).

The LNG-IUS (Mirena, Leiras Oy, Turku, Finland) is a potentially minimally-invasive surgical technique used in the treatment of menorrhagia. Furthermore, in addition to being economic, effective and reversible in terms of fertility (4), this simple procedure can be performed clinically and does not require analgesics. TBA (ThermaChoice, Gynecare Inc., Menlo Park, CA., USA) is a novel ablative tool that requires less advanced surgical skills, similar to those required for the insertion of an intrauterine contraceptive device (5). TBA is considered to be a valid and effective minimally invasive surgical alternative to hysterectomy (6).

In terms of their clinical efficacy, previous studies have compared the LNG-IUS and TBA; however, a consensus on which treatment approach is superior has yet to be reached. Four trials have determined the LNG-IUS to be more effective in reducing HMB for at least 1 year (7-10); however, another 
study demonstrated there was minimal difference between the two groups with regards to menstrual scores (11). In addition, a previous study reported that TBA appeared to be the more efficient method in the reduction of pictorial blood loss assessment chart (PBAC) scores, as compared with the LNG-IUS (12).

The main objective of the present meta-analysis was to include all of the available data from previous RCTs comparing TBA with the LNG-IUS in the treatment of HMB, including parameters such as: The patients' acceptance, the efficacy, cost effectiveness, and side effects of the two treatment options.

\section{Materials and methods}

Ethics statement. The present meta-analysis was conducted in accordance with the guidelines set out by the Preferred Reporting Items for Systematic Reviews and Meta-Analysis Statement issued in 2009 (Checklist S1) (13). All findings were evaluated according to the Grading of Recommendations Assessment, Development and Evaluation (GRADE) system (14).

Literature search and selection criteria. Relevant studies were identified using the following electronic databases: PubMed (http://www.ncbi.nlm.nih.gov/pubmed/), EMBASE (http://www.embase.com/search), the Cochrane Library (http://www.cochranelibrary.com/), Google Scholar (http://scholar.google.com/), the Chinese Scientific Journals Database (http://cstj.cqvip.com/), and the China National Knowledge Infrastructure (http://www.cnki.net/). Search terms included: "levonorgestrel intrauterine system"; "levonorgestrel intrauterine device"; "thermal balloon ablation"; "heavy menstrual bleeding"; "menorrhagia" and "randomized controlled trials." To identify additional potentially eligible studies, manual searches were conducted by reviewing textbooks, review articles and the reference lists of retrieved studies. Eligibility was not limited according to the year of publication, whereas eligible languages were restricted to English and Chinese. The literature search was independently conducted by two authors, with any discrepancies resolved in discussion with the third author. The authors or principal investigators were contacted for original information, where necessary.

Inclusion and exclusion criteria. All RCTs that compared the use of TBA with the LNG-IUS in patients with HMB were retrieved. To be considered eligible for inclusion in the present meta-analysis, the respective RCTs had to include: i) At least one of the outcome measures outlined; ii) data points presented as the mean \pm standard deviation (SD); iii) all continuous data presented as the median and/or range, with original information gathered by contacting the corresponding authors; iv) patients who had failed or refused to undertake other regular medical treatments; and v) a follow-up appointment within 3-24 months.

Potentially eligible RCTs were excluded from the present meta-analysis if: i) They included patients that currently received any form of hormone therapy or had previously undergone a hysterectomy; ii) all data were presented as the median and/or range, and there was no possibility of obtaining the original information by contacting the corresponding

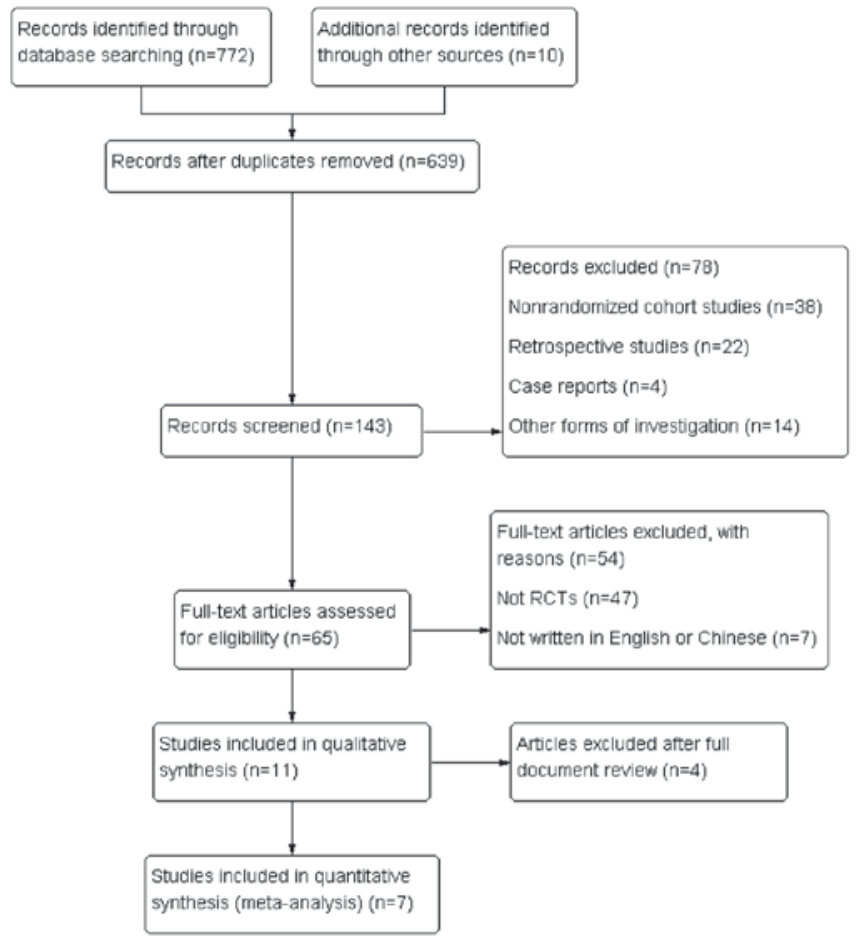

Figure 1. Flow diagram of study identification, inclusion, and exclusion.

authors; or iii) the patients displayed any additional intrauterine pathology. The final RCTs selected were as follows: Barrington et al (11), Brown et al (17), Busfield et al (9), Li et al (8), Shaw et al (7), Soysal et al (12) and Tam et al (18).

Outcomemeasures.Objectiveanalyses focusing on the following outcome variables were undertaken: i) Menstrual blood loss reduction at 3, 6, 12 and 24 months, as evaluated by PBAC scores; ii) increases in hemoglobin levels at 6 and 12 months; iii) amenorrhea rates at 3,6, 12 and 24 months; iv) overall quality of life, as measured by the Short Form-36 (SF-36) (15); v) total cost; and (vi) discontinuation rates at 3, 6, 12 and 24 months.

Data extraction. A pre-designed data extraction table was used by independent reviewers to extract the characteristic data from the eligible articles. Discrepancies were resolved through discussion or, if required, consultation with another author. In the event that RCTs presented outcomes in an unfavorable format; attempts were made to contact the authors for the original information. Data regarding the primary author's name, time of publication, study design, study population, length of follow-up, and outcomes were extracted (Table I). The study quality of the RCTs was evaluated according to the method for RCTs, as described in the Cochrane Reviewer's Handbook 5.0 (16).

Assessment of risk of bias. All RCTs included in the present meta-analysis were assessed for the following risks of bias according to the criteria outlined in the Cochrane Handbook for Systematic Reviews of Interventions: Random sequence generation (selection bias), allocation concealment (selection bias), blinding of participants and personnel (performance bias), blinding of outcome assessment (detection bias), 


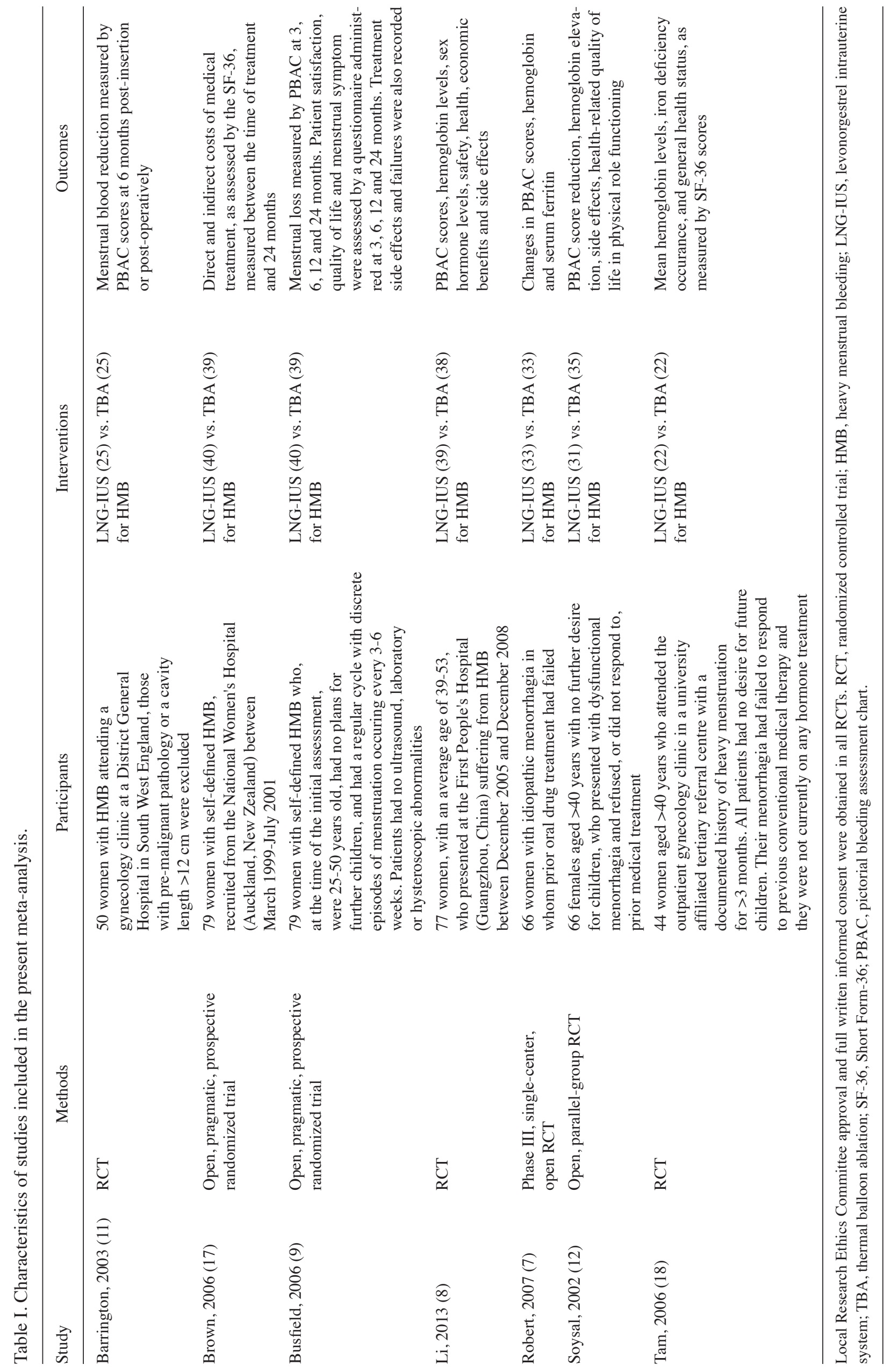


Table II. Quality assessment of the included studies using Grading of Recommendations, Assessment, Development and Evaluations analysis.

\begin{tabular}{|c|c|c|c|c|c|c|c|c|}
\hline $\begin{array}{l}\text { Outcome } \\
\text { measure }\end{array}$ & $\begin{array}{l}\text { Subjects } \\
\text { (studies) }\end{array}$ & $\begin{array}{l}\text { Risk } \\
\text { of bias }\end{array}$ & Inconsistency & Indirectness & Imprecision & $\begin{array}{c}\text { Other } \\
\text { considerations }\end{array}$ & $\begin{array}{l}\text { Overall } \\
\text { quality }^{\mathrm{d}}\end{array}$ & Importance \\
\hline \multicolumn{9}{|l|}{ PBAC scores } \\
\hline 3 months & $152(2)$ & Serious $^{\mathrm{a}, \mathrm{b}}$ & Serious $^{\mathrm{c}}$ & Not serious & Not serious & Undetected & Low & Critical \\
\hline 6 months & $186(3)$ & Serious $^{\mathrm{a}, \mathrm{b}}$ & Serious $^{\mathrm{c}}$ & Not serious & Not serious & Undetected & Low & Critical \\
\hline 12 months & $206(3)$ & Serious $^{\mathrm{a}, \mathrm{b}}$ & Serious $^{\mathrm{c}}$ & Not serious & Not serious & Undetected & Low & Critical \\
\hline 24 months & $118(2)$ & Serious ${ }^{\mathrm{a}, \mathrm{b}}$ & Not serious & Not serious & Not serious & Undetected & Moderate & Critical \\
\hline \multicolumn{9}{|l|}{ Hb levels } \\
\hline 6 months & $118(2)$ & Serious ${ }^{\mathrm{a}, \mathrm{b}}$ & Not serious & Not serious & Not serious & Undetected & Moderate & Critical \\
\hline 12 months & $163(3)$ & Serious $^{\mathrm{a}, \mathrm{b}}$ & Serious $^{c}$ & Not serious & Not serious & Undetected & Low & Critical \\
\hline \multicolumn{9}{|c|}{ Amenorrhea rate } \\
\hline 3 months & $145(2)$ & Serious $\mathrm{s}^{\mathrm{a}, \mathrm{b}}$ & Not serious & Not serious & Not serious & Undetected & Moderate & Important \\
\hline 6 months & $187(3)$ & Serious $^{\mathrm{a}, \mathrm{b}}$ & Not serious & Not serious & Not serious & Undetected & Moderate & Important \\
\hline 12 months & $223(4)$ & Serious ${ }^{\mathrm{a}, \mathrm{b}}$ & Not serious & Not serious & Not serious & Undetected & Moderate & Important \\
\hline 24 months & $131(2)$ & Serious ${ }^{\mathrm{a}, \mathrm{b}}$ & Not serious & Not serious & Not serious & Undetected & Moderate & Important \\
\hline \multicolumn{9}{|c|}{ Discontinuation rate } \\
\hline 3 months & $298(4)$ & Serious ${ }^{\mathrm{a}, \mathrm{b}}$ & Not serious & Not serious & Not serious & Undetected & Moderate & Important \\
\hline 6 months & $190(3)$ & Serious ${ }^{\mathrm{a}, \mathrm{b}}$ & Not serious & Not serious & Not serious & Undetected & Moderate & Important \\
\hline 12 months & $298(4)$ & Serious ${ }^{\mathrm{a}, \mathrm{b}}$ & Not serious & Not serious & Not serious & Undetected & Moderate & Important \\
\hline 24 months & $298(4)$ & Serious $^{\mathrm{a}, \mathrm{b}}$ & Serious $^{\mathrm{c}}$ & Not serious & Not serious & Undetected & Low & Important \\
\hline \multicolumn{9}{|c|}{ Quality of life } \\
\hline SF scores & $135(2)$ & Serious ${ }^{\mathrm{a}, \mathrm{b}}$ & Not serious & Not serious & Not serious & Undetected & Moderate & Important \\
\hline \multicolumn{9}{|l|}{ Economics } \\
\hline Total cost & $67(1)$ & Serious ${ }^{\mathrm{a}, \mathrm{b}}$ & Not serious & Not serious & Not serious & Undetected & Moderate & Important \\
\hline
\end{tabular}

${ }^{a}$ Insufficient information regarding allocation concealment; ${ }^{\mathrm{b}} \mathrm{Blinding}$ was not adequate; ${ }^{\mathrm{c}} \mathrm{I}^{2}>50 \%$; ${ }^{\mathrm{d}} \mathrm{High}$ quality, further research is very unlikely to change our confidence in the estimate of effect; moderate quality, further research is likely to have an important impact on our confidence in the estimate of effect and may change the estimate; low quality, further research is very likely to have an important impact on our confidence in the estimate of effect and is likely to change the estimate; very low quality, we are very uncertain about the estimate.

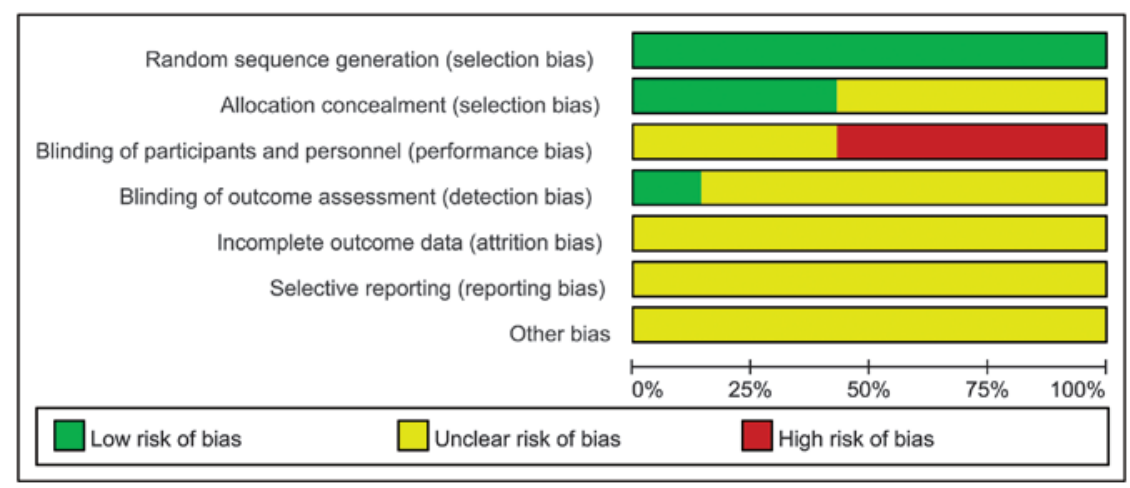

Figure 2. Risk of bias of all seven studies included study, presented as percentages.

incomplete outcome data (attrition bias) and selective reporting (reporting bias). Furthermore, the GRADE system was used to evaluate the evidence levels of the present meta-analysis, as recommended by the Cochrane Collaboration. Accordingly, the outcome measures for the LNG-IUS or TBA were graded as either: Very low, low, moderate, or high.

Statistical analysis. Data were analyzed using RevMan 5.2 software (The Nordic Cochrane Center, Copenhagen, Denmark) to conduct the meta-analysis. The relative risk (RR) with the corresponding $95 \%$ confidence interval (CI) was utilized for dichotomous data; whereas mean differences (MDs) or standardized mean differences (SMDs) with 95\% CIs were used for continuous data. Pooled estimates were calculated using the fixed-effect (Mantel-Haenszel test) or the random-effect (DerSimonian-Laird method) models. A random-effect model was applied when significant heterogeneity was found between the studies $(\mathrm{P}<0.10 ; \mathrm{I} 2>50 \%)$; otherwise, a fixed-effect model 


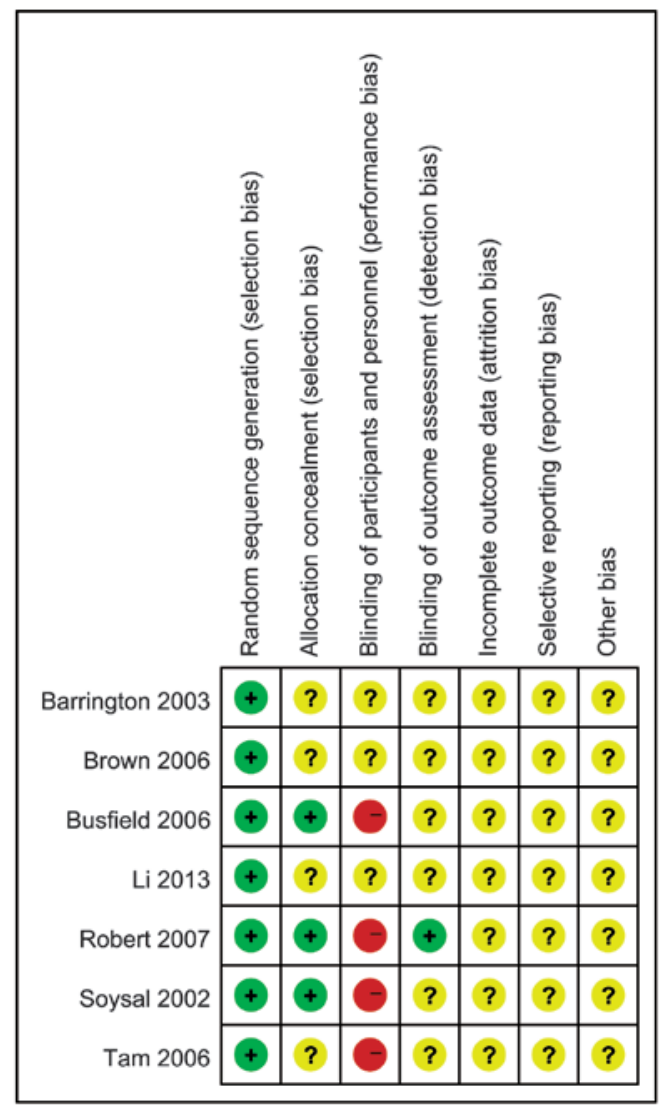

Figure 3. Risk of bias of each included study. Green, low risk; yellow, unclear risk; red, high risk.

was used. $\mathrm{P}<0.05$ was considered to indicate a statistically significant difference.

\section{Results}

Literature search. Studies were selected as outlined in Fig. 1. A total of 782 records were identified following comprehensive searches. However, the majority of these studies were non-randomized cohort studies, retrospective studies, case reports, or other forms of investigation that did not meet our inclusion criteria. Consequently, 639 studies were excluded by removing duplicate literature and through review of citations Abstracts from 143 articles were reviewed and an additional 78 trials were excluded, permitting 65 reviews for full publication review. A total of $11 \mathrm{RCTs}$ were considered appropriate for analysis (7-12,17-21). However, one of the 11 studies was subsequently excluded as the patients included all possessed large myomas (19). In addition, three further studies were excluded for the following reasons: Data was not presented as mean \pm SD (10), and we were unable to contact the corresponding author; only a 5 year follow-up was reported (20); and the LNG-IUS was compared to endometrial ablation rather than TBA (21).

Quality assessment. The remaining seven articles reported the details of random-sequence generation using computer-generated randomization $(7-9,11,12,17,18)$. Three trials reported allocation concealment with a sealed envelope $(7,9,12)$; however, concealment of allocation was deemed to be an unclear risk in the additional studies, as it was not reported. Single or double blinding was not possible in any of the studies. The risks of bias are summarized in Figs. 2 and 3.

The outcomes of the RCTs were analyzed and rated using a GRADEprofiler (http://www.gradeworkinggroup. org/society/index.htm) and some limitations were determined concerning the study design, execution, and the inconsistency of results; whereas no obvious indirectness or imprecision was detected. The quality of each outcome from the studies examined is shown in Table II.

Outcomesand synthesis ofresults.Fourstudies, which compared the PBAC scores between the respective LNG-IUS and TBA groups, were included in the present meta-analysis $(8,9,11,12)$. As outlined in Fig. 4A, the PBAC scores were divided into four subgroups $(3,6,12$ and 24 months). There were no significant differences in PBAC scores determined between the two groups at 3 months (SMD, 0.11; 95\% CI, -0.65-0.86; $\mathrm{P}=0.79), 6$ months (SMD, $-0.10 ; 95 \% \mathrm{CI},-0.54-0.33 ; \mathrm{P}=0.65)$, or 12 months (SMD, 0.41; 95\% CI, -1.47-2.28; $\mathrm{P}=0.67$ ). However, there was a significant difference in PBAC scores following 24 months of treatment (SMD, -0.86; 95\% CI, -1.22 to $-0.50 ; \mathrm{P}<0.00001)$. Statistical heterogeneity was determined among the studies $\left(\chi^{2}=101.09, \mathrm{I}^{2}=91 \% ; \mathrm{P}<0.00001\right)$; therefore a random-effect model was used. At the 24 month follow-up, patients in the LNG-IUS group demonstrated improved clinical benefit and increased control of blood loss, as compared with that of the TBA group. No significant differences were found between the two treatment groups at 3,6 and 12 months.

The hemoglobin levels of the subjects were divided into two subgroups (6 and 12 months) dictated by the period of time that elapsed between treatment and the collection of the $\mathrm{Hb}$ data $(7,8,12,18)$. As notable statistical heterogeneity was observed among the subgroups $\left(\chi^{2}=16.01, \mathrm{I}^{2}=75 \% ; \mathrm{P}=0.003\right)$, a random-effect model was used. Meta-analysis of the increased $\mathrm{Hb}$ values demonstrated no significant differences between the LNG-IUS and TBA groups at 6 months (SMD, -0.17; 95\% CI, $-0.58-0.25 ; \mathrm{P}=0.43$ ) or 12 months (SMD, -0.04; 95\% CI, -0.88-0.79; $\mathrm{P}=0.92$ ) (Fig. 4B).

Patients in both the LNG-IUS and the TBA groups suffered with the side effect of amenorrhea; therefore, a meta-analysis was conducted to compare the outcomes of the two groups $(7,9,11,18)$. The data were subsequently divided into four follow-up subgroups (3, 6, 12 and 24 months) At 3 months, a significant difference was detected (RR, 0.29; 95\% CI, 0.12-0.73; $\mathrm{P}=0.009)$; whereas at 12 and 24 months, the opposite was determined (RR, 1.65; 95\% CI, 1.15-2.38; $\mathrm{P}=0.007$ and $\mathrm{RR}, 2.49 ; 95 \% \mathrm{CI}, 1.46-4.25 ; \mathrm{P}=0.0008$, respectively). No significant difference was detected at the 6 month follow-up (RR, 0.78; 95\% CI, 0.37-1.65; P=0.51). As no significant heterogeneity was found between the subgroups, a fixed-effect model was applied to evaluate the statistics $\left(\mathrm{I}^{2}<50 \%\right)$. Thus, the authors of the present study concluded that, 3 months after treatment, patients in the TBA group experienced greater amenorrhea, as compared with those in the LNG-IUS group. The results were adverse at 12 and 24 months and no significant difference was determined between the two groups after 6 months (Fig. 5).

The overall quality of life of the patients was reported in two studies, as measured by SF-36 scores $(9,17)$. As demonstrated 

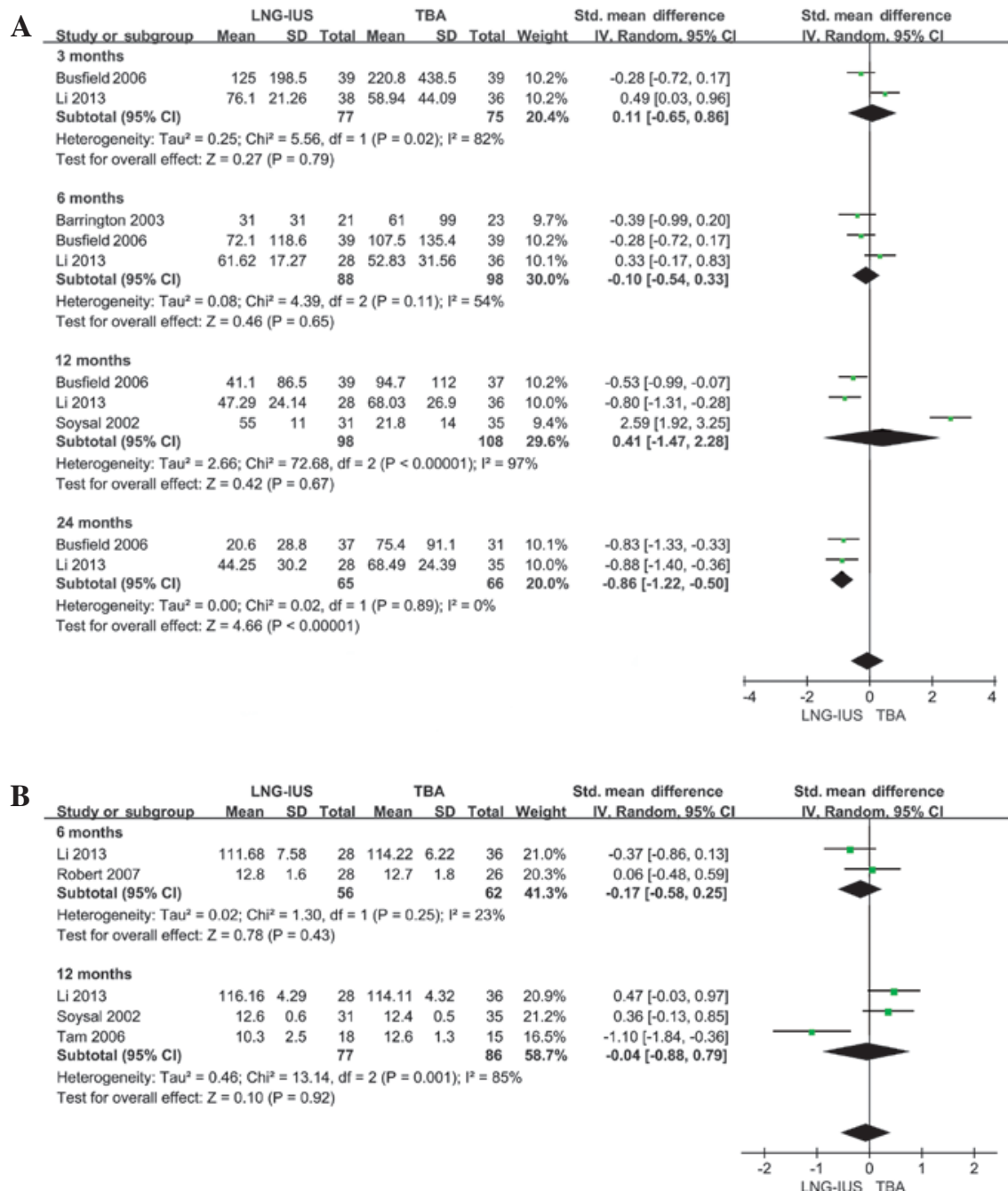

Figure 4. Forest plots of (A) the pictorial blood loss assessment chart (PBAC) scores and (B) the hemoglobin levels determined from the included studies. Data are presented as the standardized mean difference with 95\% confidence intervals (CI). LNG-IUS, levonorgestrel intrauterine system; TBA, thermal balloon ablation; SD, standard deviation.

in Fig. 6, subsequent analysis of these studies determined that there were no significant differences in the SF-36 scores between the groups (MD, 3.77; 95\% CI, -1.84-9.38; $\mathrm{P}=0.19$ ). A fixed-effect model was applied as there was no obvious statistical heterogeneity $\left(\chi^{2}=0.10, \mathrm{I}^{2}=0 \% ; \mathrm{P}=0.76\right)$.

Data concerning the total cost of treatment between the groups was supplied in one study (17) and, as exhibited in Fig. 7, TBA treatment was considerably more expensive, as compared with the LNG-IUS treatment (SMD, -2.35; 95\% CI, -2.98 to $-1.72 ; \mathrm{P}<0.00001)$.

Discontinuation of treatment rates from five studies $(7-9,11,17)$ were analyzed using a fixed-effect model, as no notable statistical heterogeneity was detected $\left(\chi^{2}=18.21\right.$, $\mathrm{I}^{2}=23 \%$; $\left.\mathrm{P}=0.20\right)$. Fig. 8 presents the comparison between the LNG-IUS and TBA groups following 3 months of treatment, and no significant difference in the discontinuation rate was detected $(\mathrm{RR}, 2.07 ; 95 \% \mathrm{CI}, 0.59-7.24 ; \mathrm{P}=0.26)$. However, following 6 and 12 months of treatment, the TBA group showed a substantially lower discontinuation rate, as compared with the LNG-IUS group at both the 6 month (RR, 3.49; 95\% CI, 1.35-9.01; $\mathrm{P}=0.01$ ) and 12 month follow-ups (RR, 2.60; 95\% CI, 1.36-4.99; $\mathrm{P}=0.004)$. However, after
24 months, no statistical disparity was observed (RR, 1.30; 95\% CI, 0.84-2.00; $\mathrm{P}=0.24)$, consistent with the findings at the 3 month follow-up.

\section{Discussion}

HMB is one of the most common gynecological disorders affecting women of reproductive age, accounting for $20 \%$ of all gynecological visits to general practitioners (11). HMB is associated with a lower quality of life, loss of productivity, and increased healthcare expenses $(22,23)$ and in usual practice, it is initially treated pharmacologically (12), with tranexamic acid and norethisterone believed to be the most effective (12). Surgical treatment for HMB often follows unsuccessful or ineffective medical therapy; however hysterectomy is a major surgical procedure with significant physical and emotional complications, in addition to the social and economic cost. Various minimally invasive surgical techniques, such as TBA, the LNG-IUS, transcervical resection of the endometrium, microwave ablation, diffused laser energy ablation, bipolar impedance-controlled ablation, cryoablation, hot saline instillation $(24,25)$, and various methods of endometrial ablation 


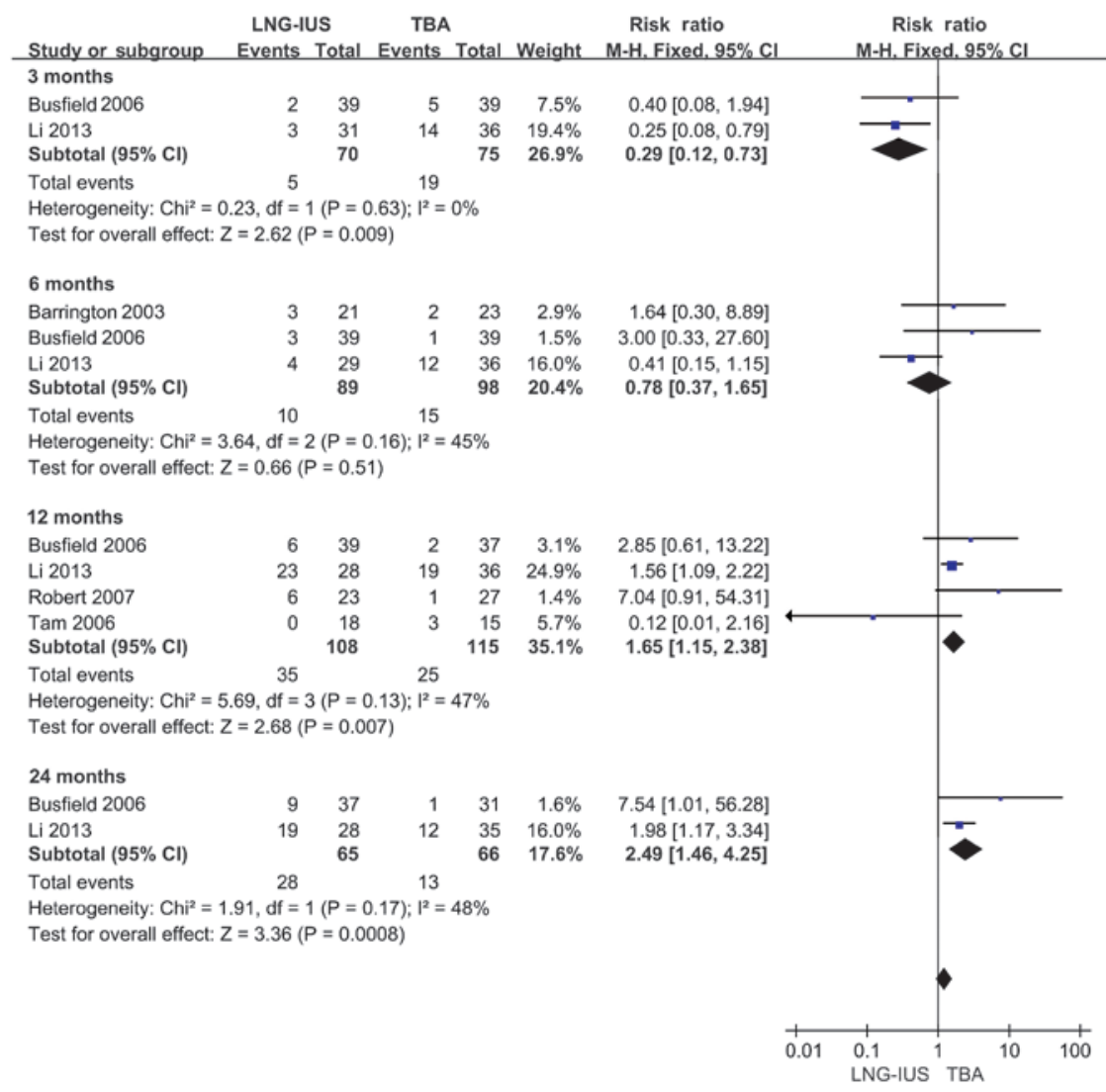

Figure 5. Forest plots of the risk ratio and 95\% confidence intervals (CI) for the amenorrhea rates. LNG-IUS, levonorgestrel intrauterine system; TBA, thermal balloon ablation.

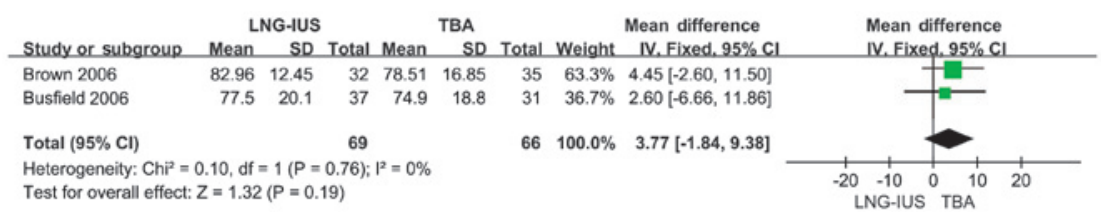

Figure 6. Forest plots of the mean difference and 95\% confidence intervals (CI) for the Short Form-36 scores. LNG-IUS, levonorgestrel intrauterine system; TBA, thermal balloon ablation; SD, standard deviation.

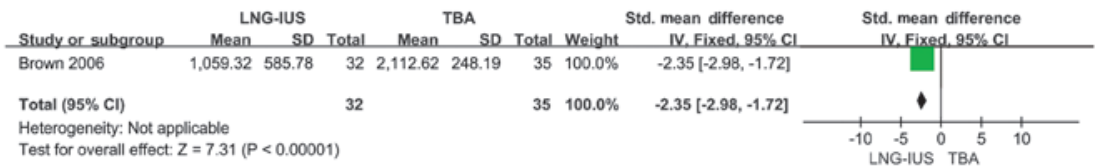

Figure 7. Forest plots of the standardized mean difference and 95\% confidence intervals (CI) for the total cost of the respective treatments. LNG-IUS, levonorgestrel intrauterine system; TBA, thermal balloon ablation; SD, standard deviation.

have been developed, with the purpose of improving menstrual symptoms, and these have achieved great success.

In 1994, TBA was initially described as a technically simple, minimally invasive surgical alternative to hysterectomy almost free of intraoperative complications (5). The main advantage of this novel technique was that treatment could be carried out under local anesthetic. Previous studies have demonstrated that TBA is as effective as endometrial resection (6) and is superior to hysteroscopic techniques in the treatment of dysfunctional menorrhagia (26). The LNG-IUD was developed in Finland during the 1980s, and is an intrauterine device that releases $20 \mu \mathrm{g}$ levonorgestrel every $24 \mathrm{~h}$ over 5 years. Although initially licensed as a contraceptive, in 1990 the LNG-IUS was tested and reported to be effective in the treatment of menorrhagia, as a non-contraceptive benefit (27) and LNG-IUS is reported to have $>4$ million users worldwide (28). Previous controlled trials and case studies have demonstrated significant reductions in menstrual blood loss (74-97\%) following treatment with the LNG-IUS (29), and the LNG-IUS has been widely accepted by patients with HMB due to its simplicity, reversibility, and relatively low complication rate.

The present meta-analysis compared the efficacy and safety of TBA with the LNG-IUS using primary and secondary 


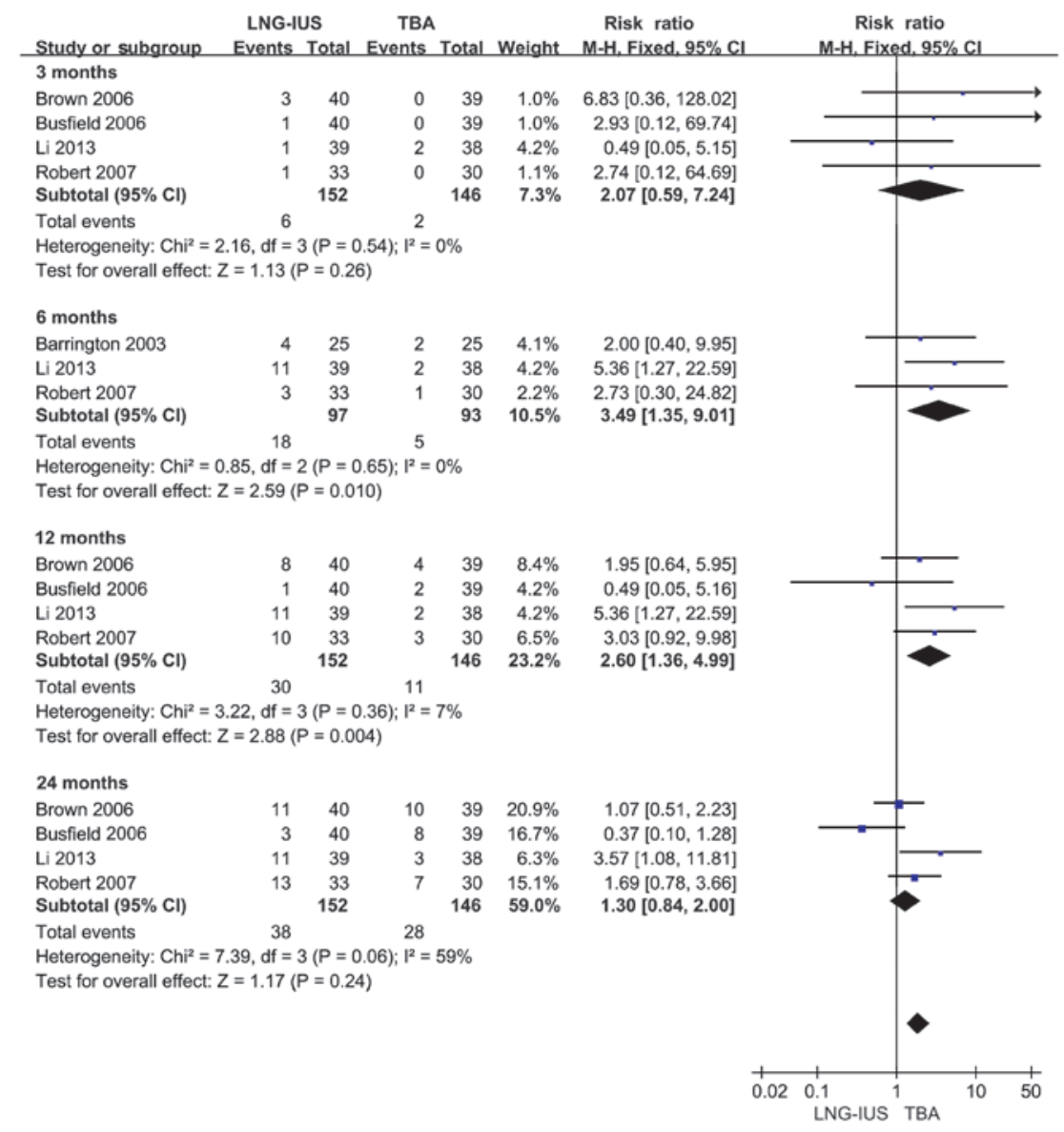

Figure 8. Forest plots of the risk ratio and the 95\% confidence interval (CI) for the discontinuation rates. LNG-IUS, levonorgestrel intrauterine system; TBA, thermal balloon ablation.

outcome measures. Menstrual blood loss, quantified using PBAC scores, was considered the most significant outcome. Four RCTs were analyzed with regards to PBAC in the present study, and following 24 months of treatment with the LNG-IUS, $50 \%$ reported significantly decreased PBAC scores as compared with TBA $(8,9,11,12)$. Two studies $(8,9)$ demonstrated a decrease in PBAC scores following 12 months of treatment with either the LNG-IUS or TBA, whereas another study (12) reported the opposite result and the present meta-analysis established that there was no significant difference between the LNG-IUS and TBA. Furthermore, an increase in hemoglobin levels demonstrated the effectiveness of TBA and the LNG-IUS, and the present meta-analysis concluded that there were no differences between the two treatments.

Following TBA or LNG-IUS treatment, numerous complications may occur, such as: Irregular bleeding, persistent spotting, amenorrhea, mastalgia, weight gain, mood swings, bloating, acne, greasy skin, nausea, headache, reduced libido, leg pain, dysmenorrhea, and lower abdominal pain. In order to compare the subsequent amenorrhea rates following treatment with either the LNG-IUS or TBA, five RCTs $(7-9,11,17)$ were pooled and, following 12 and 24 months of treatment, the present meta-analysis demonstrated that the TBA group suffered considerably less amenorrhea, as compared with the LNG-IUS group. However, this outcome was different following 3 months of treatment. When evaluating the discontinuation rate between the LNG-IUS and TBA groups, the present meta-analysis determined that, following 6 and 12 months of treatment, a greater number of patients who were treated with the LNG-IUS withdrew, as compared with those in the TBA group. However, no differences were observed following 3 and 24 months of treatment.

The present meta-analysis also evaluated the secondary outcome measures, such as the cost of treatment and patients' quality of life, between the two treatments. One RCT (17) compared the total treatment cost between the LNG-IUS and TBA groups, the results of which determined that the LNG-IUS was more cost-effective, as compared with TBA, for the treatment of HMB. The quality of life of the patients was measured using SF-36 scores pooled from two RCTs $(9,17)$, and no significant difference was observed between the LNG-IUS and the TBA groups.

The present meta-analysis of RCTs compared the clinical efficacy, safety, cost-effectiveness, and acceptance of the LNG-IUS and TBA in patients suffering from HMB, and the results may suggest that, as a long-term treatment therapy, the LNG-IUS is of greater benefit to patients than TBA. Although the LNG-IUS is more likely to be associated with long-term amenorrhea, it is more efficient in reducing patient blood loss (PBAC scores) and more cost-effective compared with TBA. Therefore, LNG-IUS has advantages over TBA as a long-term treatment. Regarding amenorrhea, as the LNG-IUS is a reversible device placed in the uterus, it may be reversible for patient's menstrual period following treatment. At the 
24 month follow-up appointment, the total cost of treatment and the PBAC scores were significantly decreased in patients treated with the LNG-IUS. However, the patients' quality of life, measured by the SF-36 scores, and hemoglobin levels were not significantly different between the two treatment groups. For relatively short term treatment (6-12 months) TBA was determined to be more acceptable to patients as the discontinuation rate within the TBA group was decreased, as compared with that of the LNG-IUS group. However, for long-term treatment (24 months), no difference was detected between the groups, and this may suggest that patients became equally acceptable to these two methods; however, following 12 and 24 months of treatment, the most significant side effect, amenorrhea, occurred in a greater number of patients who were treated with the LNG-IUS. Therefore, the present study may provide gynecologists with an evidence-based strategy for choosing between treatments for HMB.

Although a conclusion was achieved in the present study, the following three possible limitations should be considered: i) The present meta-analysis was limited to seven studies with 467 subjects, and the sample size was not large enough to draw a substantial conclusion; ii) significant heterogeneity may have remained due to the complication of subjective or objective factors such as study design, methodology, follow-up period, and outcome variables; therefore, to alleviate the heterogeneity and determine a conservative estimate, a random-effect model was employed. iii) Funnel plots evaluating the risk of publication bias were not attempted as there were only a limited number of studies and the results may have been unreliable.

In conclusion, the present meta-analysis provided preliminary evidence that, for the treatment of patients with HMB, the LNG-IUS may be superior to TBA with regards to a reduction in PBAC scores following long-term treatment, and the total cost of treatment. This evidence may be useful to gynecologists in choosing a therapy for patients with HMB. Nevertheless, further more well-designed RCTs are required to explore the differences between the LNG-IUS and TBA.

\section{Acknowledgements}

The authors of the present study gratefully acknowledge the manuscript editing support of the highly qualified editors at American Journal Experts.

\section{References}

1. Nilsson L and Rybo G: Treatment of menorrhagia. Am J Obstet Gynecol 110: 713-720, 1971.

2. Maresh MJ, Metcalfe MA, McPherson K, Overton C, Hall V, Hargreaves J, Bridgman S, Dobbins J and Casbard A: The VALUE national hysterectomy study: Description of the patients and their surgery. BJOG 109: 302-312, 2002.

3. Stovall DW: Alternatives to hysterectomy: Focus on global endometrial ablation, uterine fibroid embolization, and magnetic resonance-guided focused ultrasound. Menopause 18: 437-444, 2011.

4. Middleton LJ, Champaneria R, Daniels JP, Bhattacharya S, Cooper KG, Hilken NH, O'Donovan P, Gannon M, Gray R, Khan KS, et al; International Heavy Menstrual Bleeding Individual Patient Data Meta-analysis Collaborative Group: Hysterectomy, endometrial destruction, and levonorgestrel releasing intrauterine system (Mirena) for heavy menstrual bleeding: Systematic review and meta-analysis of data from individual patients. BMJ 341: c3929, 2010.
5. Neuwirth RS, Duran AA, Singer A, MacDonald R and Bolduc L: The endometrial ablator: A new instrument. Obstet Gynecol 83: 792-796, 1994.

6. Loffer FD: Three-year comparison of thermal balloon and rollerball ablation in treatment of menorrhagia. J Am Assoc Gynecol Laparosc 8: 48-54, 2001.

7. Shaw RW, Symonds IM, Tamizian O, Chaplain J and Mukhopadhyay S: Randomised comparative trial of thermal balloon ablation and levonorgestrel intrauterine system in patients with idiopathic menorrhagia. Aust N Z J Obstet Gynaecol 47: 335-340, 2007.

8. Li M, Kang JL, Ji YQ, Liu HF, Nie ML and Wang XX: Effect of levonorgestrel-releasing intrauterine system and thermal balloon ablation for the treatment of heavy menstrual bleeding. Bei Jing Da Xue Zhong Gyo Fu Chan Ke Lin Chuang Za Zhi 6: 501-505, 2013 (In Chinese).

9. Busfield RA, Farquhar CM, Sowter MC, Lethaby A, Sprecher M, $\mathrm{Yu}$ Y, Sadler LC, Brown P and Johnson N: A randomised trial comparing the levonorgestrel intrauterine system and thermal balloon ablation for heavy menstrual bleeding. BJOG 113: 257-263, 2006.

10. de Souza SS, Camargos AF, de Rezende CP, Pereira FA, Araújo CA and Silva Filho AL: A randomized prospective trial comparing the levonorgestrel-releasing intrauterine system with thermal balloon ablation for the treatment of heavy menstrual bleeding. Contraception 81: 226-231, 2010.

11. Barrington JW, Arunkalaivanan AS and Abdel-Fattah M: Comparison between the levonorgestrel intrauterine system (LNG-IUS) and thermal balloon ablation in the treatment of menorrhagia. Eur J Obstet Gynecol Reprod Biol 108: 72-74, 2003.

12. Soysal M, Soysal S and Ozer S: A randomized controlled trial of levonorgestrel releasing IUD and thermal balloon ablation in the treatment of menorrhagia. Zentralbl Gynakol 124: 213-219, 2002.

13. Moher D, Liberati A, Tetzlaff J and Altman DG; PRISMA Group: Preferred reporting items for systematic reviews and meta-analyses: The PRISMA statement. Ann Intern Med 151: 264-269, 2009.

14. Atkins D, Best D, Briss PA, Eccles M, Falck-Ytter Y, Flottorp S, Guyatt GH, Harbour RT, Haugh MC, Henry D, et al; GRADE Working Group: Grading quality of evidence and strength of recommendations. BMJ 328: 1490, 2004.

15. Hays RD, Sherbourne CD and Mazel RM: The RAND 36-Item Health Survey 1.0. Health Econ 2: 217-227, 1993.

16. Higgins JPT and Green S (eds): Cochrane Handbook for Systematic Reviews of Interventions (Version. 5.1.0). The Cochrane Collaboration, Oxford, England 2011. http://handbook. cochrane.org. Accessed Dec 30, 2012.

17. Brown PM, Farquhar CM, Lethaby A, Sadler LC and Johnson NP: Cost-effectiveness analysis of levonorgestrel intrauterine system and thermal balloon ablation for heavy menstrual bleeding. BJOG 113: 797-803, 2006.

18. Tam WH, Yuen PM, Shan Ng DP, Leung PL, Lok IH and Rogers MS: Health status function after treatment with thermal balloon endometrial ablation and levonorgestrel intrauterine system for idiopathic menorrhagia: A randomized study. Gynecol Obstet Invest 62: 84-88, 2006.

19. Kim JY, No JH, Kim K, Kim YB, Jee BC, Lee JR and Suh CS: Effect of myoma size on failure of thermal balloon ablation or levonorgestrel releasing intrauterine system treatment in women with menorrhagia. Obstet Gynecol Sci 56: 36-40, 2013.

20. Silva-Filho AL, Pereira Fde A, de Souza SS, Loures LF, Rocha AP, Valadares CN, Carneiro MM, Tavares RL and Camargos AF: Five-year follow-up of levonorgestrel-releasing intrauterine system versus thermal balloon ablation for the treatment of heavy menstrual bleeding: a randomized controlled trial. Contraception 87: 409-415, 2013.

21. Herman MC, van den Brink MJ, Geomini PM, van Meurs HS, Huirne JA, Eising HP, Timmermans A, Pijnenborg JM, Klinkert ER, Coppus SF, et al: Levonorgestrel releasing intrauterine system (Mirena) versus endometrial ablation (Novasure) in women with heavy menstrual bleeding: A multicentre randomised controlled trial. BMC Womens Health 13: 32, 2013.

22. Barnard K, Frayne SM, Skinner KM and Sullivan LM: Health status among women with menstrual symptoms. J Womens Health (Larchmt) 12: 911-919, 2003.

23. Frick KD, Clark MA, Steinwachs DM, Langenberg P, Stovall D, Munro MG and Dickersin K; STOP-DUB Research Group: Financial and quality-of-life burden of dysfunctional uterine bleeding among women agreeing to obtain surgical treatment. Womens Health Issues 19: 70-78, 2009. 
24. Köchli OR: Endometrial ablation in the year 2000 - do we have more methods than indications? Contrib Gynecol Obstet 20: 91-120, 2000.

25. Sowter MC: New surgical treatments for menorrhagia. Lancet 361 1456-1458, 2003.

26. Grainger DA, Tjaden BL, Rowland C and Meyer WR: Thermal balloon and rollerball ablation to treat menorrhagia: Two-year results of a multicenter, prospective, randomized, clinical trial. J Am Assoc Gynecol Laparosc 7: 175-179, 2000.

27. Andersson JK and Rybo G: Levonorgestrel-releasing intrauterine device in the treatment of menorrhagia. Br J Obstet Gynaecol 97: 690-694, 1990
28. Hurskainen R, Teperi J, Rissanen P, Aalto AM, Grenman S, Kivelä A, Kujansuu E, Vuorma S, Yliskoski M and Paavonen J: Clinical outcomes and costs with the levonorgestrel-releasing intrauterine system or hysterectomy for treatment of menorrhagia: Randomized trial 5-year follow-up. JAMA 291: 1456-1463, 2004.

29. Stewart A, Cummins C, Gold L, Jordan R and Phillips W: The effectiveness of the levonorgestrel-releasing intrauterine system in menorrhagia: A systematic review. BJOG 108: 74-86, 2001. 